\title{
The Conformal Window in SU(3) Yang-Mills
}

\section{Ethan T. Neil*}

Sloane Physics Laboratory, Yale University, New Haven, CT 06511, United States

E-mail: ethan.neileyale.edu

\section{Thomas Appelquist}

Sloane Physics Laboratory, Yale University, New Haven, CT 06511, United States

E-mail: thomas.appelquist@yale.edu

\section{George T. Fleming}

Sloane Physics Laboratory, Yale University, New Haven, CT 06511, United States

E-mail: george.fleming@yale.edu

Yang-Mills theory is known to exhibit very different behavior as the number of light fermion flavors $N_{f}$ is varied. In particular, for sufficiently large $N_{f}$ the theory changes fro confining to conformal behavior in the infrared. We have obtained results for SU(3) Yang-Mills, based on the study of the running coupling through the Schrödinger Functional approach, that the transition between these two phases occurs between 8 and 12 flavors. I will present the details of our staggered fermion simulations at $N_{f}=8$ and 12 .

The XXVI International Symposium on Lattice Field Theory

July 14 - 19, 2008

Williamsburg, Virginia, USA

\footnotetext{
* Speaker.
} 


\section{Introduction}

With the Large Hadron Collider (LHC) about to come online, the bulk of particle theory research is focused on extending the Standard Model to the TeV-scale energies which will soon become experimentally accessible. A number of the theoretical models considered to describe LHC physics involve new strong dynamics, i.e. new interactions resembling the only known strong force, QCD. Models such as technicolor [1, 2], composite Higgs [3] and topcolor [4] are just a few examples of Standard Model extensions involving new strong interactions.

If another strongly interacting theory does appear in nature, lattice gauge theory will provide the ideal way to study it. Perturbation theory can provide only a limited understanding of a strongly coupled theory, and no other non-perturbative method is as mature and broadly applicable as lattice simulation. Even if no strongly coupled dynamics are revealed at the LHC, the study of more exotic Yang-Mills theories can be interesting in and of itself. Mapping out the parameter space of general Yang-Mills theories by varying the number of colors and light flavors would lead to a more solid theoretical understanding, which might open up new avenues of non-perturbative investigation into QCD or any strongly-coupled theory that might occur beyond the LHC.

It is also possible that more exotic behavior may arise from strong dynamics. In particular, it is known that $\mathrm{SU}(N)$ gauge theory with enough light fermion flavors $N_{f}$ develops an infrared conformal fixed point $[5,6]$. Although not as directly applicable to LHC physics as the above examples, these theories are also of interest in the context of research into conformal field theory, e.g. $[7,8]$. Although not truly conformal, these theories do show approximate scale invariance, and the ability to simulate such a theory on the lattice may offer new ways to study conformal behavior non-perturbatively. Furthermore, quantities derived from our running coupling measurement, such as the fixed-point coupling strength and the anomalous scaling dimension at the fixed point, may be of interest to model builders thinking about conformal behavior.

Two of the most important qualities of QCD ( $N_{f}=2$ at relatively low energies) are asymptotic freedom (the coupling strength vanishes at high energy/short distance) and confinement (the coupling strength diverges at low energy/long distance, i.e. there are no free states with color charge.) These properties are strongly dependent on $N_{f}$; it is well known that for $N_{f}>16.5$, SU(3) Yang-Mills is no longer asymptotically free [9]. These theories are too exotic for our current purposes. For $N_{f}$ just below this critical value, the short-distance theory still resembles QCD, but in the infrared the coupling flows to a perturbative fixed point [6] - confinement is lost.

Clearly as we decrease the number of fermions, at some critical point $2<N_{f}^{c}<16.5$ a transition must take place from a fixed-point theory to a confining theory. Since the theory for $N_{f}>N_{f}^{c}$ shows conformal behavior in the infrared, we refer to the range $N_{f}^{c}<N_{f}<16.5$ as the conformal window. Although perturbation theory is useful near the top of the conformal window, as we decrease $N_{f}$ the strength of the fixed-point coupling increases, eventually making the perturbative expansion useless. $N_{f}^{c}$ is thus unknown, and non-perturbative study is essential to accurately determine its value and the nature of the transition into the conformal window.

\section{General approach and methods}

As noted above, the two phases on either side of the transition at $N_{f}^{c}$ are characterized by 

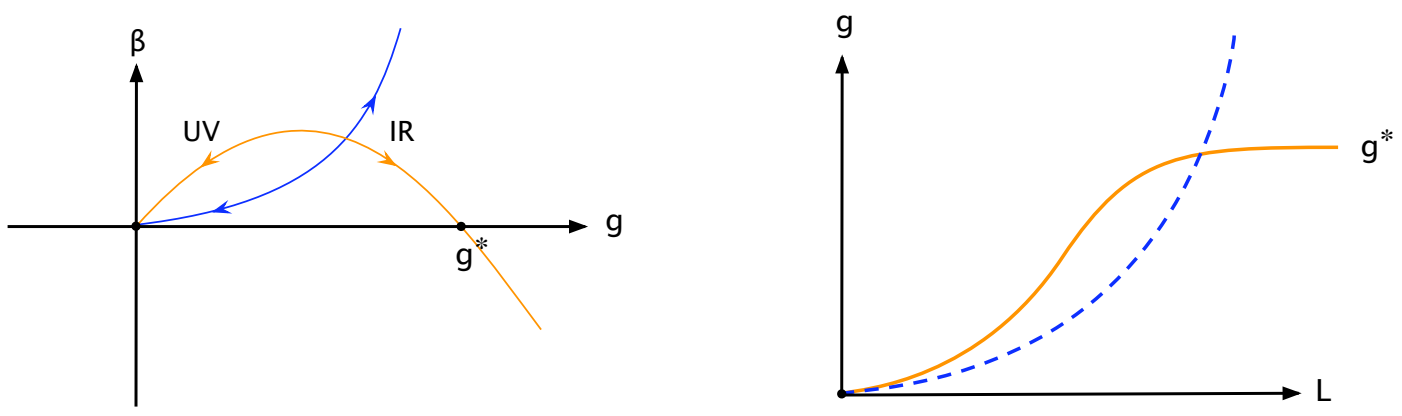

Figure 1: Cartoon showing the phases inside and outside of the conformal window, in terms of the running coupling. The $\beta$-function (left) vanishes in the ultraviolet in both cases, showing asymptotic freedom. The difference is seen in the infrared: a QCD-like theory confines, with the $\beta$-function appearing to diverge in the IR, while a theory in the conformal window develops an infrared fixed point at $g=g^{\star}$. This is visible in the running coupling itself (right) as a plateau.

confining and conformal behavior in the infrared; a simple way to distinguish them is thus to measure the running coupling of the theory, and search for the presence or absence of an infrared fixed point. This will be the focus of our lattice simulations.

More concretely, we begin with the $\beta$-function of the theory,

$$
\beta(g) \equiv L \frac{d g^{2}}{d L}=b_{0} g^{4}+b_{1} g^{6}+b_{2} g^{8}+\cdots
$$

which encapsulates the evolution of the coupling constant. The first two coefficients in the perturbative expansion above are independent of renormalization scheme, and given by

$$
\begin{aligned}
& b_{0}=\frac{2}{(4 \pi)^{2}}\left(11-\frac{2}{3} N_{f}\right) \\
& b_{1}=\frac{2}{(4 \pi)^{4}}\left(102-\frac{38}{3} N_{f}\right) .
\end{aligned}
$$

Asymptotic freedom is maintained so long as $b_{0}$ is positive, which holds for any $N_{f}$ below 16.5. However, just below this value of $N_{f}$, the second coefficient $b_{1}$ is negative. Thus in the two-loop $\beta$-function, a zero develops, which the coupling constant will run towards in the infrared - an $I R$ fixed point. In two-loop perturbation theory this occurs for $N_{f}$ near 8 , but the value of the coupling at the predicted fixed point is very strong; non-perturbative study is the only way to truly determine $N_{f}^{c}$. The direct non-perturbative measurement of the evolution of the coupling constant is a simple way to distinguish these two phases. A sketch of the $\beta$-function and coupling constant evolution in the above cases is shown in figure 2.

We choose to employ the Schrödinger Functional (SF) definition of the running coupling in our measurement. We work in a box of size $L^{4}$; the scale of the box $L$ is thus the only scale in the system, and our coupling will be defined at this same scale. Periodic boundary conditions are taken in the spatial directions, while Dirichlet boundary conditions in Euclidean time will be used 


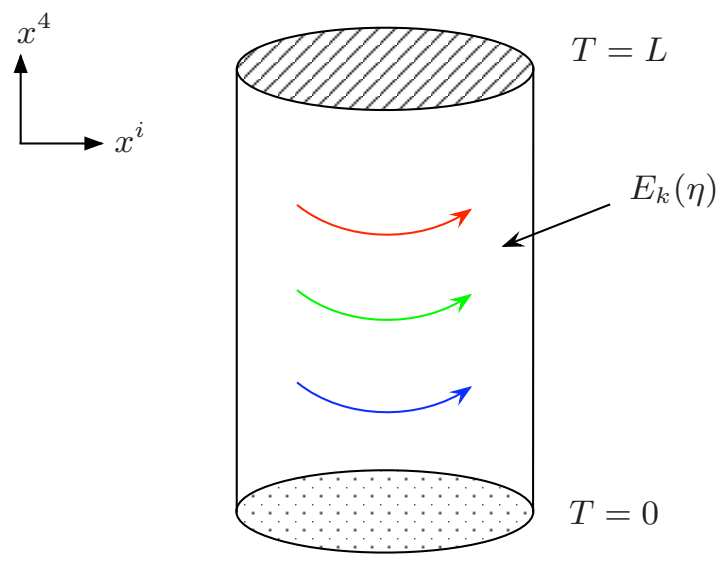

Figure 2: Sketch of the Schrödinger Functional setup. Dirichlet boundary conditions in the Euclidean time direction are set to impose a constant background chromoelectric field.

to impose a constant chromoelectric background field. Measuring the response of the system to variations in the background field will define the coupling strength. In particular, for the running coupling measurement the boundary gauge field values are set to be consistent with the classical solution for a constant chromoelectric background field, with strength parameterized by a dimensionless value $\eta$. The coupling is then measured to be inversely proportional to the response of the action as the strength parameter $\eta$ is varied,

$$
\frac{d S}{d \eta} \equiv \frac{k}{\bar{g}^{2}}
$$

where $k$ is a constant of proportionality, which is set so that the observable $\bar{g}^{2}$ matches onto the perturbative running coupling. See figure 2 for a sketch of the overall setup.

\section{Lattice simulation details}

From a lattice simulation point of view, the SF approach provides an attractive alternative to the standard Wilson loop method for measuring the running coupling strength, one which is free from finite-size effects since the coupling is measured directly at the scale of the box, $L$. The presence of the box also lifts the zero modes of the Dirac operator, making it possible to simulate directly at zero fermion mass [10]. This is crucial, since non-zero fermion masses would occlude the infrared behavior of the theory below that mass scale as the fermions are screened out.

Simulations are carried out using the MILC code [11], version 6, with some customization. Evolution of the gauge configurations is accomplished using the hybrid molecular dynamics (HMD) technique, with the $\mathrm{R}$ algorithm used to incorporate the fermions [12]. Trajectories are taken to be unit length, and the step size $\Delta \tau$ of the MD integrator is varied. This approach is subject to numerical integration errors of order $(\Delta \tau)^{2}$, which are generally found to be negligible at small $L / a$; on larger lattices, extrapolation to $\Delta \tau=0$ is performed to eliminate these errors.

We use the staggered fermion action [13], which is relatively cheap and simple to simulate with at zero fermion mass. However, due to the geometry of staggered fermions in combination with 
the presence of Dirichlet boundary conditions in time instead of periodic, this forces us to simulate on lattices with even $L / a$ and odd $T / a$. With $T=L \pm a$, an $O(a)$ lattice artifact is introduced when we attempt to measure the running coupling at the scale $L$. This artifact is removed explicitly by simulating at both $T=L \pm a$ and averaging over the two [13].

Since each gauge configuration yields only a single measurement of the running coupling, there is no volume self-averaging, and extracting a measurement with a reasonable error requires a substantial number of configurations. Furthermore, very long autocorrelation times are noted, on the order of thousands of MD trajectories in the worst cases; as the updating of gauge fields is done locally while our observable is measured on the scale of the entire box $L$, it is unsurprising that many updates are required to create statistically independent measurements of $\bar{g}^{2}(L)$. We collect 20,000 to $80,000 \mathrm{MD}$ trajectories at each value of $\beta$, each box size $L / a$ and integrator each step size $\Delta \tau$, in order to remove statistical bias and extract a good estimate of the running coupling.

A given lattice simulation must be performed at a fixed bare lattice coupling $g_{0}^{2}$, which in turn fixes the scale of the lattice spacing $a$. Variation of the box size $L / a$ with $a$ fixed does not yield enough evolution of scale to get an accurate picture of the running of the coupling constant; practically we cannot increase $L / a$ beyond 20 without the cost of the simulation becoming prohibitive, whereas we would like to measure the evolution of the coupling over many $e$-foldings.

To circumvent this problem, we use a systematic procedure known as step scaling to link together the results of many simulations with various scales $a$, and extract the correct continuum running. We define the lattice step scaling function to be

$$
\Sigma\left(s, \bar{g}^{2}(L), a / L\right)=\bar{g}^{2}(s L)+O(a / L)
$$

We will fix the step size $s=2$ from here forward. In practice, after a starting value $\bar{g}^{2}(L)$ has been selected, there is a tuning step in which several lattices with different $a / L$ are adjusted to yield the same measurement $\bar{g}^{2}(L)$. Keeping $a$ fixed, $a / L$ is then doubled on each lattice, and the measurement of the coupling on this larger lattice is exactly $\Sigma\left(2, \bar{g}^{2}(L), a / L\right)$. We can then extrapolate $a / L \rightarrow 0$ to recover the continuum value of $\bar{g}^{2}(2 L)$. Iterating this procedure allows us to step from $\bar{g}^{2}(L) \rightarrow \bar{g}^{2}(2 L) \rightarrow \cdots$ indefinitely, capturing the evolution of the coupling constant over very large changes in scale. We determine $\Sigma$ at four distinct values of $a / L$, stepping from $4 \rightarrow 8,6 \rightarrow 12,8 \rightarrow 16$, and $10 \rightarrow 20$.

Naïvely, staggered fermions possess discretization errors of $O\left(a^{2}\right)$, so one would expect a quadratic extrapolation in $a / L$ to best fit the measured values for $\Sigma(2, u, a / L)$. However, other methods can be attempted. In particular, dropping the $4 \rightarrow 8$ step is well-motivated by arguing that the lattice artifacts on the $L / a=4$ lattices are extremely large, to the point of non-analyticity; on the smallest $\left(4^{3} \times 3\right)$ lattice, $O(a)$ boundary artifact operators which "stick out" from the Dirichlet boundaries will actually overlap. The remaining three values of $\Sigma(2, u, a / L)$ are well fit by a pure constant extrapolation, i.e. a weighted average. The difference in choice of extrapolation method is found to be the dominant source of systematic error in our simulations; therefore these two extrapolation methods (quadratic to all four values of $\Sigma(2, u, a / L)$ and constant dropping $L / a=4$ ) are taken to define the edges of our systematic error band.

Results are depicted in figure 3 . The $N_{f}=8$ theory looks consistent with the confining phase, showing no evidence of turnover to an infrared fixed point. The $N_{f}=12$ theory, however, shows 

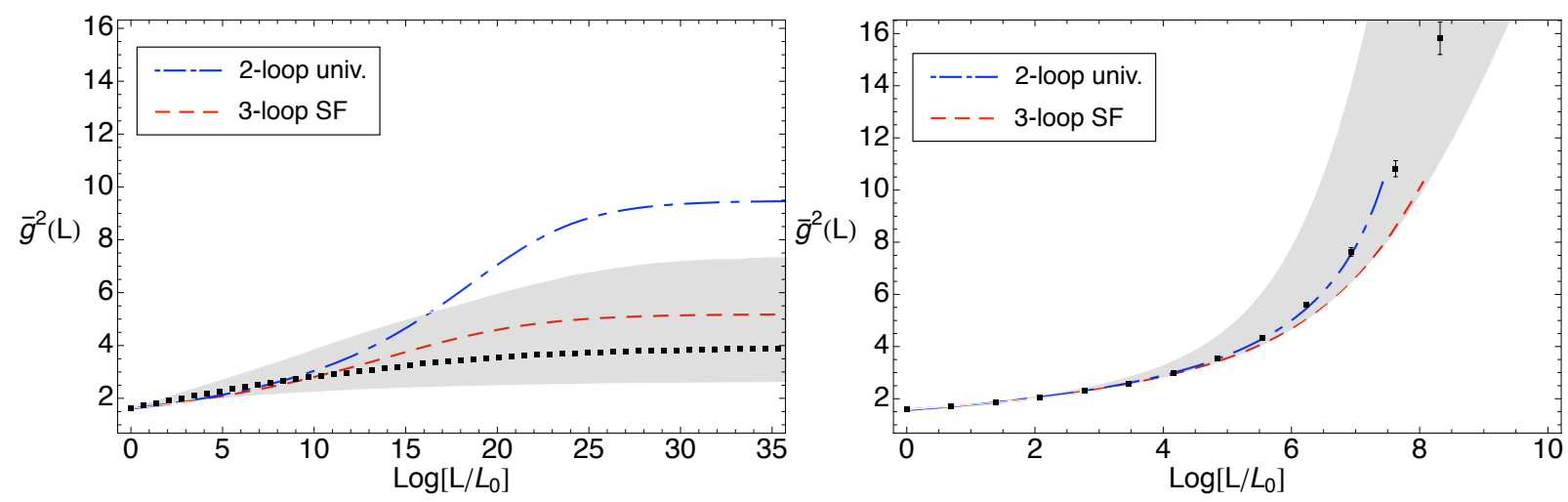

Figure 3: Running coupling at $N_{f}=12$ (left) and $N_{f}=8$ (right) in $S U$ (3) Yang-Mills, as in [14]. The systematic error band (grey) is determined by variation of continuum extrapolation method, as described in the text. The lack of an observed fixed point at $N_{f}=8$ indicates that this value of $N_{f}$ lies outside the conformal window.

clear evidence of an IRFP. Indeed, this theory represents the first non-perturbative measurement of conformal behavior outside of supersymmetry.

Work is still ongoing to refine these simulations and their analysis. The systematic error bands shown are quite large, and reducing them would increase confidence in our results. In particular at $N_{f}=12$, a reduced error band should admit a measurement of the anomalous dimension of the coupling constant as it approaches the fixed point, a quantity which may interest model-builders working with conformal and near-conformal theories.

\section{Conclusion and outlook}

In conclusion, we have shown evidence that the lower boundary of the conformal window $N_{f}^{c}$ lies between 8 and 12 flavors. This is in agreement with most perturbative estimates, e.g. [15-19], and in contrast with the previous lattice results of Iwasaki et al. [20].

Furthermore, the results as depicted are consistent with 3-loop perturbation theory, and indeed the coupling strength of the IR fixed point observed at $N_{f}=12$ is quite weak. If this continues to be the case as the error band is narrowed, it would lend strength to the claim by Gardi et al. that perturbation theory is valid all the way to the bottom of the conformal window [16].

The logical next step is a similar measurement of the running coupling at $N_{f}=10$, which is ongoing. To continue simulating directly at zero quark mass, we must switch to Wilson fermions; use of staggered fermions at $N_{f}=10$ would require taking a fractional power of the fermion determinant, which can be problematic if the fermion mass is set to zero [21].

Wilson fermions are inherently much more expensive to simulate with than staggered, but this will be offset in several ways. First of all, simulation at odd $L / a$ is possible with Wilson fermions; this will allow us to add more values of $\Sigma(2, u, a / L)$ to the continuum extrapolation, constraining it better. We will also attempt to reduce $a / L$ dependence by using the clover-improved action, and perturbative counterterms for boundary $O(a)$ operators. Additionally, we can switch to the Wilsonoriented Chroma code base, which is very optimized and offers improved algorithms like rational hybrid Monte Carlo, which should further diminish simulation cost. 


\section{References}

[1] Steven Weinberg. Implications of dynamical symmetry breaking. Phys. Rev. D, 13:974-996, 1976.

[2] Edward Farhi and Leonard Susskind. Technicolor. Phys. Rept., 74:277, 1981.

[3] David B. Kaplan, Howard Georgi, and Savas Dimopoulos. Composite higgs scalars. Phys. Lett. B, page $187,1984$.

[4] D. A. Dicus, Bhaskar Dutta, and S. Nandi. Top quark signature in extended color theories. Phys. Rev. D, 51:6085-6091, 1995 .

[5] William E. Caswell. Asymptotic Behavior of Nonabelian Gauge Theories to Two Loop Order. Phys. Rev. Lett., 33:244, 1974.

[6] Tom Banks and A. Zaks. On the phase structure of vector-like gauge theories with massless fermions. Nuclear Physics B, 196:189, 1982.

[7] Markus A. Luty and Takemichi Okui. Conformal technicolor. JHEP, 09:070, 2006.

[8] M. Ibe, Y. Nakayama, and T. T. Yanagida. Conformal gauge mediation. Phys. Lett. B, 649:292-298, 2007.

[9] D. J. Gross and Frank Wilczek. Asymptotically free gauge theories. 1. Phys. Rev. D, 8:3633-3652, 1973.

[10] Rainer Sommer. Non-perturbative qcd: renormalization, $o(a)$-improvement and matching to heavy quark effective theory. 2006.

[11] Carleton DeTar et al. http://www.physics.utah.edu/ detar/milc/, Sep 2002.

[12] Steven A. Gottlieb, W. Liu, D. Toussaint, R. L. Renken, and R. L. Sugar. Hybrid molecular dynamics algorithms for the numerical simulation of quantum chromodynamics. Phys. Rev., D35:2531-2542, 1987.

[13] Urs M. Heller. The Schrödinger functional running coupling with staggered fermions. Nucl. Phys., B504:435-458, 1997.

[14] Thomas Appelquist, George T. Fleming, and Ethan T. Neil. Lattice Study of the Conformal Window in QCD-like Theories. Phys. Rev. Lett., 100:171607, 2008.

[15] Thomas Appelquist, Andrew G. Cohen, and Martin Schmaltz. A new constraint on strongly coupled field theories. Phys. Rev., D60:045003, 1999.

[16] Einan Gardi and Georges Grunberg. The conformal window in QCD and supersymmetric QCD. JHEP, 03:024, 1999.

[17] Thomas Appelquist, John Terning, and L. C. R. Wijewardhana. Postmodern technicolor. Phys. Rev. Lett., 79:2767-2770, 1997.

[18] V. A. Miransky and Koichi Yamawaki. Conformal phase transition in gauge theories. Phys. Rev., D55:5051-5066, 1997.

[19] Masafumi Kurachi and Robert Shrock. Study of the change from walking to non-walking behavior in a vectorial gauge theory as a function of N(f). JHEP, 12:034, 2006.

[20] Y. Iwasaki, K. Kanaya, S. Kaya, S. Sakai, and T. Yoshie. Phase structure of lattice QCD for general number of flavors. Phys. Rev., D69:014507, 2004.

[21] Stephen R. Sharpe. Rooted staggered fermions: Good, bad or ugly? PoS, LAT2006:022, 2006. 